\title{
ANXA11 mutations prevail in Chinese ALS patients with and without cognitive dementia
}

Kang Zhang, PhD, * Qing Liu, MD, PhD, * Keqiang Liu, PhD, Dongchao Shen, PhD, Hongfei Tai, PhD, Shi Shu, MD, PhD, Qingyun Ding, MD, Hanhui Fu, PhD, Shuangwu Liu, PhD, Zhili Wang, PhD, Xiaoguang Li, MD,

Mingsheng Liu, MD, Xue Zhang, MD, PhD, and Liying Cui, MD, PhD

Neurol Genet 2018;4:e237. doi:10.1212/NXG.0000000000000237

\section{Abstract}

\section{Objective}

To investigate the genetic contribution of ANXA11, a gene associated with amyotrophic lateral sclerosis (ALS), in Chinese ALS patients with and without cognitive dementia.

\section{Methods}

Sequencing all the coding exons of ANXA11 and intron-exon boundaries in 18 familial amyotrophic lateral sclerosis (FALS), 353 unrelated sporadic amyotrophic lateral sclerosis (SALS), and 12 Chinese patients with ALS-frontotemporal lobar dementia (ALS-FTD). The transcripts in peripheral blood generated from a splicing mutation were examined by reverse transcriptase PCR.

\section{Results}

We identified 6 nonsynonymous heterozygous mutations ( 5 novel and 1 recurrent), 1 splice site mutation, and 1 deletion of 10 amino acids (not accounted in the mutant frequency) in 11 unrelated patients, accounting for a mutant frequency of 5.6\% (1/18) in FALS, $2.3 \%(8 / 353)$ in SALS, and $8.3 \%(1 / 12)$ in ALS-FTD. The deletion of 10 amino acids was detected in 1 clinically undetermined male with an ALS family history who had atrophy in hand muscles and myotonic discharges revealed by EMG. The novel p. P36R mutation was identified in 1 FALS index, 1 patient with SALS, and 1 ALS-FTD. The splicing mutation (c.174-2A>G) caused in-frame skipping of the entire exon 6. The rest missense mutations including p.D40G, p.V128M, p.S229R, p.R302C and p.G491R were found in 6 unrelated patients with SALS.

\section{Conclusions}

The ANXA11 gene is one of the most frequently mutated genes in Chinese patients with SALS. A canonical splice site mutation leading to skipping of the entire exon 6 further supports the loss-of-function mechanism. In addition, the study findings further expand the ANXA11 phenotype, first highlighting its pathogenic role in ALS-FTD.

\author{
Correspondence \\ Dr. X. Zhang \\ xuezhang@pumc.edu.cn or Dr. Cui \\ pumchcuily@yahoo.com
}

From the Department of Neurology and Laboratory of Clinical Genetics, Peking Union Medical College Hospital (K.Z., Q.L., D.S., H.T., Q.D., H.F., S.L., Z.W., X.L., M.L., X.Z., L.C.) and McKusick-Zhang Center for Genetic Medicine (K.L., S.S. X.Z.), State Key Laboratory of Medical Molecular Biology, Institute of Basic Medical Sciences Chinese Academy of Medical Sciences, School of Basic Medicine, Peking Union Medical College; and Neuroscience Center (K.Z., Q.L., K.L., D.S., H.T., S.S., Q.D., H.F., S.L., Z.W., X.L., M.L., X.Z., L.C.), Chinese Academy of Medical Sciences, Beijing, China.

Funding information and disclosures are provided at the end of the article. Full disclosure form information provided by the authors is available with the full text of this article at Neurology.org/NG. 


\section{Glossary}

ALS = amyotrophic lateral sclerosis; ExAC = Exome Aggregation Consortium; FALS = familial amyotrophic lateral sclerosis; FTD = frontotemporal lobar dementia; MAF = minor allele frequency; PUMCH = Peking Union Medical College Hospital; SALS $=$ sporadic amyotrophic lateral sclerosis.

Amyotrophic lateral sclerosis (ALS) is a fatal neurologic disease characterized by progressive paralysis and ultimately respiratory failure within 5 years of symptom onset. ${ }^{1}$ Approximately $5 \%-10 \%$ of ALS cases exhibit familial amyotrophic lateral sclerosis (FALS) inheritance, and causative gene mutations can be found in $60 \%$ of patients with FALS. The remaining $90 \%-95 \%$ of ALS cases exhibit sporadic amyotrophic lateral sclerosis(SALS), and mutations in the same genes are responsible for $10 \%$ of patients with SALS. ${ }^{2}$ To date, rare variants in more than 30 genes have been reported to cause or be associated with ALS. ${ }^{3}$ Recently, mutations of ANXA11 have been identified in patients with ALS of European ancestry, but pathogenicity of ANXA11 in other ALS cohorts remained unproved. ${ }^{4}$ In the current study, we investigated ANXA11 mutations in Chinese ALS patients with or without cognitive decline.

\section{Methods}

\section{Study population}

A total of 383 Chinese patients with ALS were recruited at the ALS clinic of Neurology Department, Peking Union Medical College Hospital (PUMCH) from January 2016 to August 2017. Patients were diagnosed according to the established clinical criteria and standard protocol by specialists in ALS. ${ }^{5-7}$

\section{Mutation screening}

Genomic DNA was extracted from peripheral blood leukocytes using a QIAamp DNA Blood Midi Kit (Qiagen, Valencia, CA) according to the manufacturer's instructions. All coding exons and at least $100 \mathrm{bp}$ of flanking introns of ANXA11 (NM_145869) were amplified and put to Sanger Sequencing with published primer sequences ${ }^{4}$ using an ABI 3730 automated DNA sequencing system (Applied Biosystems). Sequence alignment were performed against human genome (UCSC hg 19) using CodonCode Aligner. Each identified mutation was reamplified and resequenced from both ends with the same primer pairs. Rare variants (minor allele frequency $[\mathrm{MAF}]<$ $0.1 \%$ ) with high conservation were further assessed for pathogenicity using online prediction software, SIFT (sift.bii.a-star.edu.sg), and PolyPhen-2 (genetics.bwh.harvard.edu/pph2/). Bioinformatics analysis software MutationTaster (mutationtaster.org), ESEfinder 3.0 (krainer01.cshl.edu/cgi-bin/tools/ ESE3/esefinder.cgi?process=home), and Human Splicing Finder 3.0 (umd.be/HSF3/index.html) were applied to predict the effect of mutation on mRNA splicing.

The current cohort of patients had been screened for known ALS genes (SOD1, ALS2, SETX, FUS, VAPB, ANG, TARDBP, FIG4, OPTN, VCP, UBQLN2, SIGMAR1, CHMP2B, PFN1,
C9ORF72, ATXN2, AR, DCTN1, NEFH, PRPH, DAO, TFG, $T A F 15$, and GRN) using a massive parallel sequencing gene panel by the Ion Torrent PGM system as described before ${ }^{8}$ and Sanger sequencing of genes including CHCHD10, TBK1, CCNF, and GLE1. C9orf72 expansion was also examined using repeat-primed PCR.

\section{Transcripts investigation of the splicing mutation}

Total RNA was extracted using TRIzol (Invitrogen) from peripheral white blood cells. Then, $2.5 \mu \mathrm{g}$ of RNA was used to perform reverse transcriptase PCR according to the Reverse Transcription System (Promega, Madison, WI) instructions. cDNA was amplified using the primer pair ANXA11-RT-F ( $5^{\prime}$-CCATGAGCTACCCTGGCTAT-3') and ANXA11-RT-R (5'-GACTCCCCAGGCAGTCAAT-3') located at ANXA11 exon 4 and exon 8 (shown in figure 3), respectively. The PCR products were isolated on a $1.5 \%$ agarose gel. DNA fragments of interest were gel purified and sequenced.

\section{Standard protocol approvals, registrations, and patient consents}

The study was approved by the Ethical Review Board of PUMCH. After an informed consent form was obtained from the participant or his family, the blood specimen of the participant was collected.

\section{Results}

The current patient cohort included 18 FALS index patients and 365 unrelated patients with SALS. Twelve patients with SALS had concomitant frontotemporal lobar dementia (ALSFTD). Of the 12 patients with ALS-FTD, 11 met the probable behavioral variant FTD (bvFTD) diagnosis following the Rascovsky criteria, ${ }^{7}$ and the remaining one was diagnosed as semantic dementia. The demographic features of the current cohort are presented in table 1 .

\section{Mutations in AXAN11 and updated mutation spectrum of ALS causal genes in Chinese patients with ALS}

In total, we identified 6 nonsynonymous heterozygous mutations (5 novel and 1 recurrent), 1 splice site mutation, and 1 deletion of 10 amino acids (not accounted in the mutant frequency) in 11 unrelated patients, accounting for a mutant frequency of $5.6 \%(1 / 18)$ in FALS, $2.3 \%(8 / 353)$ in SALS, and $8.3 \%(1 / 12)$ in ALS-FTD (figures 1 and 2 and figure e-1, links.lww.com/NXG/A57). These mutations were all absent in 384 healthy controls. No additional mutations were detected in other known ALS genes. A missense variant we identified 
Table 1 Demographics of the study population

\begin{tabular}{llll}
\hline Variable & FALS & SALS & ALS-FTD \\
\hline $\mathbf{N}$ & 18 & 353 & 12 \\
\hline Mean onset age (y) & $44.71 \pm 12.54$ & $51.92 \pm 11.56$ & $58.60 \pm 9.812$ \\
\hline Male, $\mathbf{n}(\%)$ & $10(55.6)$ & $197(55.8)$ & $8(66.7)$ \\
\hline Site of onset, bulbar (\%) & $2(11.1)$ & $48(13.6)$ & $2(16.7)$ \\
\hline
\end{tabular}

Abbreviations: ALS = amyotrophic lateral sclerosis; FALS = familial amyotrophic lateral sclerosis; FTD = frontotemporal lobe dementia; SALS = sporadic amyotrophic lateral sclerosis.

(c.119A $>$ G, p.D40G) was already reported in European patients with ALS. Three novel mutations (c.174-2A $>G$, p.A58_Q187del; c.382G $>$ A, p.V128M; and c.687T $>$ A, p.S229R) were identified, which had not been documented in online databases of human polymorphisms including dbSNP147, 1000 Genomes, and Exome Aggregation Consortium (ExAC). The remaining 3 coding variants (c.107C $>\mathrm{G}$, p.P36R; c.904C >T, p.R302C; and c.1471G >A, p.G491R) had an allele frequency of less than $0.005 \%$ in the ExAC database and were predicted to be damaging by bioinformatics analysis. Of note, the p.P36R mutation was detected both in patients with ALS and in patients with ALSFTD. Detailed information concerning the ANXA11 mutations and mutation carriers identified in the current cohort are presented in table e-1 (links.lww.com/NXG/A56). In addition, a deletion mutation (c.1146_1175del, p.L383_V392del) was also detected in 1 clinically undetermined male patient whose father was diagnosed as having ALS and deceased at the age of 70 years before DNA was obtainable. The son had mild atrophy in the left hand, which was noticed at the age of 31 years; myotonic discharges in bilateral limbs and thoracic paraspinal muscles were revealed by EMG (figure e-1).

\section{Effect of the c.174-2A>G variation on ANXA11 mRNA splicing}

The c.174-2A $>\mathrm{G}$ variation was predicted to affect splicing by multiple bioinformatics analysis. RNA extracted from the patient's peripheral blood showed the alternatively spliced transcript lacking the entire coding part of exon 6 (p.A58_Q187del). Electrophoresis on a $1.5 \%$ agarose gel revealed $\overline{1}$ fragment encompassing exons 5-7 in the healthy control. Of interest, 3 distinct fragments were observed in the mutant patient. Sanger sequencing for those 3 fragments confirmed that an approximate 750-bp transcript was a normal spliced fragment encompassing exons 5-7, an approximate 250-bp transcript was an aberrant one resulting from exon 6 skipping, and an approximate 500-bp transcript was a heterodimer consisting a normal transcript and an abnormal transcript. Therefore, the c.174-2A $>\mathrm{G}$ mutation affects exon 6 causing 58-187 amino acid deletions. The schematic diagram of gel fractionation and sequence traces of reverse transcriptase PCR products are shown in figure 3.

\section{Clinical characteristics of patients with the ANXA11 mutation}

All 10 patients ( 7 men and 3 women) carrying ANXA11 variant came from Chinese mainland, and the age of symptom onset

Figure 1 Mutations identified in ANXA11 in ALS and ALS-FTD patients

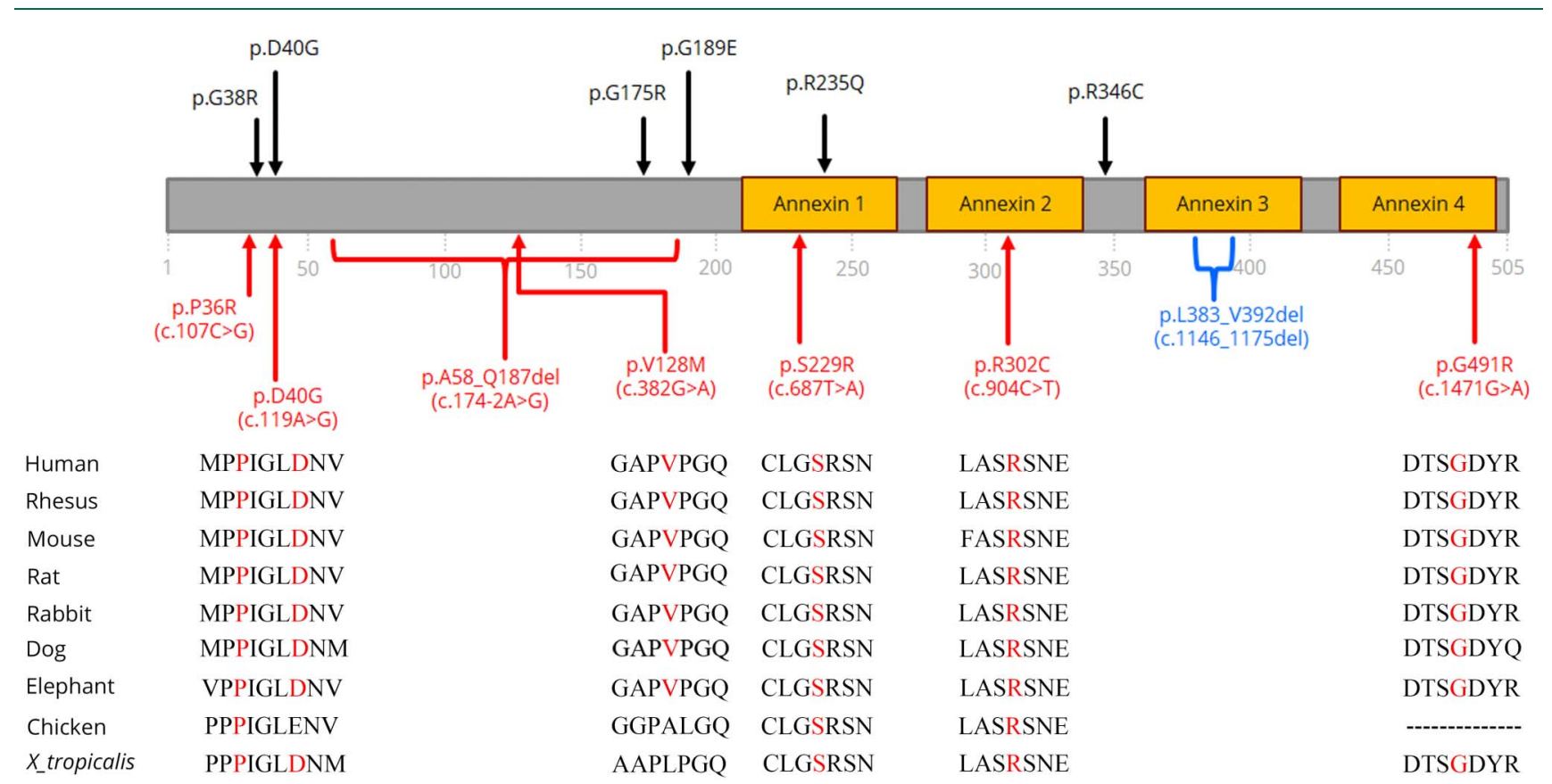

Mutations found in the previous study and in the present study are marked in black and in red, respectively. A deletion mutation associated to a clinically undetermined patient is marked in blue. Conservation of amino acid across species is shown at the bottom. ALS = amyotrophic lateral sclerosis; FTD = frontotemporal lobe dementia. 


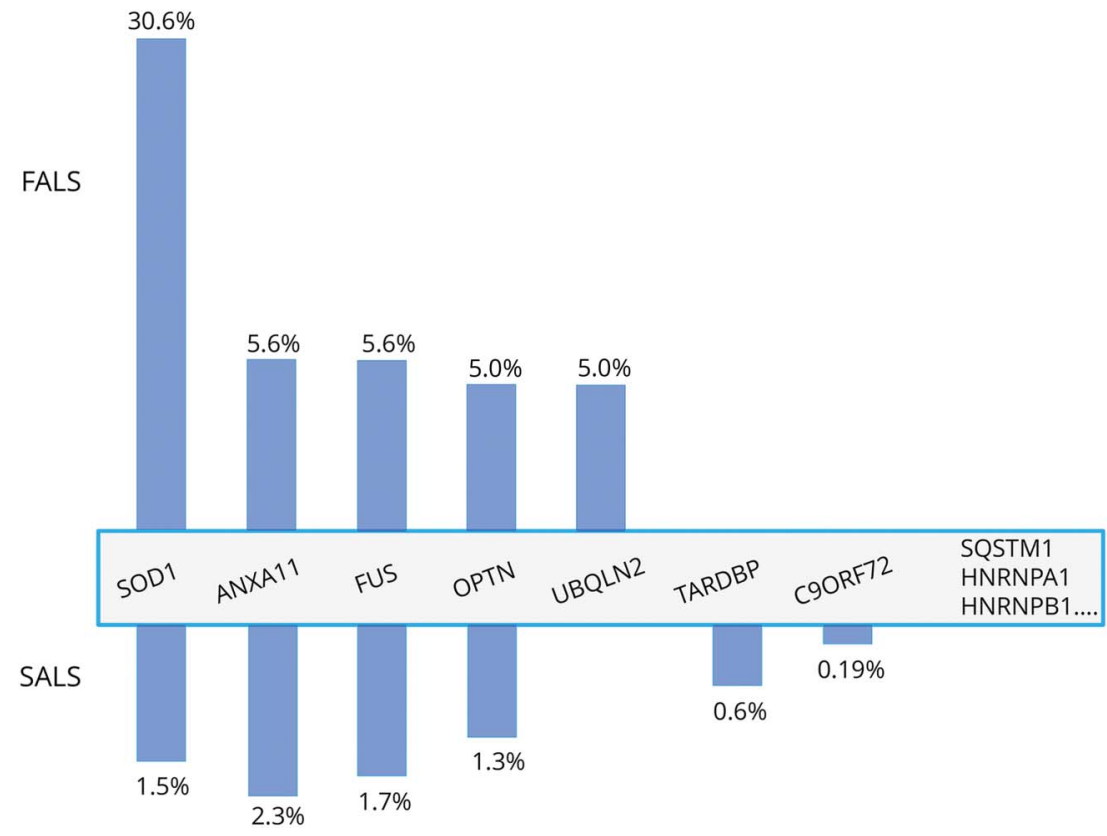

SOD1 is the most common mutant gene in FALS, and ANXA11 in SALS. FALS = familial amyotrophic lateral sclerosis; SALS = sporadic amyotrophic lateral sclerosis.

ranged from 37 to 71 years. Four patients initially exhibited dysarthria and dysphagia, and 6 patients exhibited limb weakness as the first symptom. Two patients (case 534, p.P36R, and case 395 , p.P36R) died, survived 28 and 24 months, respectively. The rest of the patients are still alive, and 2 individuals (case 479, p.G491R, and case 545, p.A58_Q187del) survived for over 5 years. All patients denied sarcoidosis. There was a patient (case 534, p.P36R) with a positive family history of ALS whose older brother had similar symptoms including dysarthria and dysphagia and died of respiratory failure 2 years after disease onset. The p.P36R mutation carrier (case 474) initially presented slurred speech at age 70 years. A few months later, his symptom quickly extended to his both legs with muscle weakness and atrophy. About a year and a half later, His family members reported that he became easily irritable, aggressive and inappropriate behaviors, such as laughing at inappropriate occasions. Over the subsequent 1 year, his both arms showed weakness. EMG demonstrated chronic and acute denervation changes in the cervical, thoracic, and lumbar segments. MRI showed bilateral temporal lobe atrophy and moderate frontal atrophy. 18F-fluorodeoxyglucosePET imaging showed bilateral frontotemporal hypometabolism. His Mini-Mental State Examination score was 20/30, and the Montreal Cognitive Assessment (MoCA) test score was 15/30. He was diagnosed as having ALS with bvFTD. In addition, case 479 (p.G491R) was accompanied by cognitive impairment. The detailed clinical information is summarized in table e-1 (links.lww.com/NXG/A56).

\section{Discussion}

With evolving technologies for gene sequencing, a large number of ALS causal genes were found. In the past 3 years, 7 causative genes, including MATR3, TUBA4A, CHCHD10, GLE1, TBK1, NEK1, and $C C N F^{3,9-16}$, were confirmed to be related to ALS. However, the frequency of mutations in these genes is very low in Chinese patients with ALS. Lately, 6 ANXA11 heterozygous missense mutations were reported in European patients with ALS. ${ }^{4}$ In the present study, we screened the ANXA11 gene mutation in a large ALS and ALS-FTD cohort of Chinese mainland for the first time. We found 7 potentially pathogenic mutations in a total of 10 unrelated cases. The ANXA11 mutation frequency in our current cohort is $2.6 \%(10 / 383)$, with $5.6 \%(1 / 18)$ in FALS, $2.3 \%(8 / 353)$ in SALS, and $8.3 \%(1 / 12)$ in ALS-FTD, which is higher than the frequency in a European cohort (1\% in FALS and $1.7 \%$ in SALS). ${ }^{4}$ A recent meta-analysis regarding Asian ALS populations revealed that the most frequent mutations were SOD1 (SALS $1.5 \%$, FALS 30.0\%), followed by FUS (SALS 0.9\%, FALS 6.4\%), C9orf72 (SALS 0.3\%, FALS 2.3\%), and TARDBP (SALS $0.2 \%$, FALS $1.5 \%$ ) mutations. ${ }^{17}$ Moreover, we previously reported the prevalence of FUS, SOD1, and OPTN responsible for $1.7 \%, 1.5 \%$, and $1.3 \%$ of Chinese patients with SALS respectively, whereas the mutation frequency in Chinese FALS was $30.6 \%$ for SOD 1 mutations, $5.6 \%$ for FUS mutations, and 5\% for OPTN mutations. ${ }^{8}$ Considering those studies together, among Chinese patients with ALS, ANXA11 is the leading gene in SALS, and SOD1 is the most frequent causal gene in FALS (figure 2), which indicate a different genetic architecture between Caucasians and Chinese. Therefore, the sequencing analysis of SOD1, ANXA11, and FUS is preferentially recommended in Chinese patients with ALS.

All variants identified except for p.D40G are first reported. The p.D40G mutation was previously reported in European 


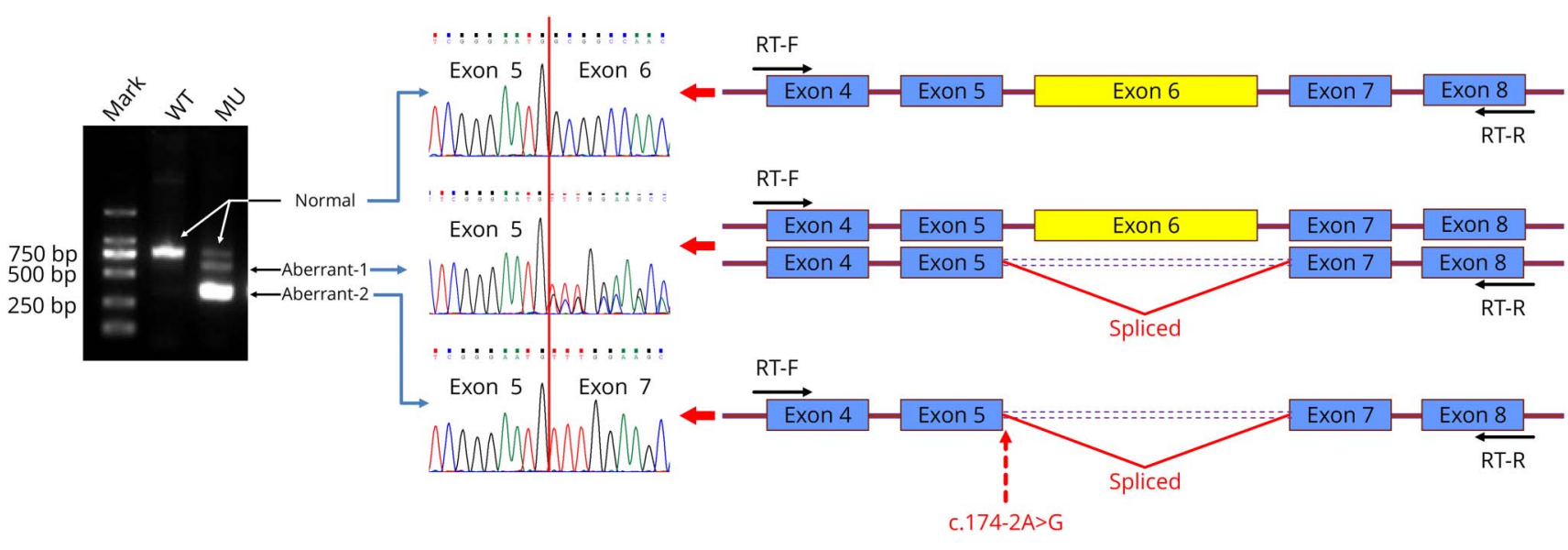

A 1.5\% agarose gel fractionation of RT-PCR products of blood RNA shows the distinct fragments of cDNA obtained by specific primers. Lane WT represents a normal transcript in a healthy control. Lane MU represents an aberrant transcript in a patient carrying the $c .174-2 A>G$ mutation, which contains 3 different bans. Sequencing results show that the band located at $750 \mathrm{bp}$ implies a normal transcript; the band located at 250 bp implies an aberrant transcript, which was caused by exon 6 skipping; and the band located at $500 \mathrm{bp}$ is confirmed to be a result of formation of heterodimer consisting of 1 normal transcript and 1 aberrant transcript. ALS = amyotrophic lateral sclerosis; cDNA = complementary DNA; FTD = frontotemporal lobe dementia; RT-PCR = reverse transcription PCR.

patients with ALS and had a common European founder. ${ }^{4}$ Among these mutations, p.P36R, p.R302C, and p.G491R had a very low allele frequency $(<0.005 \%)$ in the ExAC database. Nevertheless, the pathogenicity of the 3 mutational sites is supported by the following evidences. First, the 3 mutations were absent from our healthy controls. Second, the 3 mutant positions are all highly conserved across species, implicating that those amino acids are of functional importance. Third, different in silico prediction algorithms, all demonstrated the 3 sites pathogenic. We should recognize that many definite pathogenic variants of diseasecausing genes, such as cancer gene and cardiomyopathy gene, may have an allele frequency of less than $0.01 \%$ in the ExAC database. ${ }^{18}$ In addition, the p.V128M variant was predicted to be benign by bioinformatics software, but it was not observed in population-based databases and our controls. No mutations of other ALS causal genes were discovered in the patient carrying p.V128M. The p.V128M mutation may play a detrimental role in ALS, and further functional experiment and additional cohorts screening will draw firm conclusions.

It is difficult to pinpoint dominant features of ANXA11mutated patients. Patients carrying the same variant may have different clinical presentations. For instance, in contrast to our 1 patient (case 506) harboring the p.D40G variant initially presented left arm weakness at his 59 years, European p.D40G variant carriers exhibited late disease onset (average, 67 years) and mostly bulbar onset reported by the original ariticle. ${ }^{4}$ Similarly, the cognitive function varied among patients harboring the identical p.P36R variant, with normal cognitive ability and concomitant FTD observed in the present study. Moreover, we found that a splice site mutation c.174-2A $>\mathrm{G}$ (p.A58_Q187del) and the living affected carrier (case 545) showed slow progression and $>8$ years disease duration, which was different from other patients. These variable manifestations imply apparent clinical heterozygous among the specific ANXA11 mutation carriers. Case 660 is a p.S229R carrier of Han ethnicity, and she also harbors c.688C >T (p.R230C). The common single nucleotide polymorphism (rs1049550, C>T, p.R230C, MAF 0.44) in ANXA11 was associated with the increased risk of sarcoidosis. ${ }^{19}$ Actually, the ANXA11 rs1049550 $\mathrm{T}$ allele is a protective factor for affecting sarcoidosis in the Chinese Han population. ${ }^{20}$ It is notable that nobody had a family or personal history of sarcoidosis in our cohort. Of interest, the p.L383_V392del mutation carrier (case 399) did not fulfill the diagnosis of ALS, although his father died of the disease. This may further suggest the varied phenotype caused by the ANXA11 mutation and would require long-term followup of the patient.

In the current study, 2 unrelated patients with ALS (case 534 and case 395) harboring the p.P36R mutation had no cognitive impairment. Case 534 has a positive family history. Unfortunately, unavailability of DNA samples from his family members precluded us from confirming whether the mutation segregates with the disease. The same genetic change was also detected in an individual (case 474) having ALS-FTD and had classic upper and lower motor nervous system damage combined with bilateral frontotemporal atrophy. To our knowledge, it is the first time for us to find the ANXA11 mutation in the patient with ALS-FTD, which provides further genetic support for ALS and FTD as a disease continuum. Indeed, the number of causal ALS-FTD genes increased rapidly in the past few years. Mutations in C9orf72, TARDBP, and TBK1 are the major genetic causes in ALS-FTD, and TDP-43 inclusions as a common pathologic character are resulted from each of these variants. ${ }^{1,21-25}$ Pathogenic mutations in other genes including FUS, CHCHD10, CCNF, UBQLN2, SQSTM1, and VCP have also 
been found in patients with ALS-FTD. ${ }^{26}$ However, the genetic etiology of quite a few patients with ALS-FTD remains unclear.

ANXA11 is located on human chromosome 10q22.3 and encodes the 505 amino acid annexin A11 protein that belongs to a group of calcium-dependent phospholipidbinding proteins. Annexin A11 is a member of the annexin protein family. Four conserved annexin domains (annexin 1 , annexin 2, annexin 3, and annexin 4) constitute its conserved $\mathrm{C}$ terminus. ${ }^{27}$ Unlike other annexins, it has a unique long $\mathrm{N}$-terminal domain containing the binding site of calcyclin (residues 50-62). ${ }^{28}$ Calcyclin can mediate the ubiquitination and proteasomal degradation of many target proteins. ${ }^{29}$ Functional data showed that p.D40G and p.G38R, which both located in proximity to the calcyclin binding region, could result in abnormal binding of calcyclin. ${ }^{4}$ That may be the major cause of formation of annexin A11-positive inclusions in postmortem nervous tissue observed from ANXA11 mutant carriers. Of note, the exonskipping mutation c.174-2A>G (p.A58_Q187del) resulting in 58-187 amino acid deletions directly impaired the functional domain, undoubtedly disrupted calcyclin binding. Furthermore, the p. P36R mutation close to the residues 50-62 might affect normal calcyclin binding as well, although there is no functional study to support this possibility. Combined with original article's experimental data, ${ }^{4} 13$ of the 23 ANXA11 mutations (p.P36R, p.G38R, p.D40G, and p.A58_Q187del) are around the binding site of calcyclin. We speculate that this region is a hot spot of mutation and has significant biological functions. Those variants disrupt the normal physiologic function of annexin A11, suggesting that the pathogenesis of ANXA11 mutations is mediated by a loss-of-function mechanism. However, the pathogenetic mechanism of the remaining ANXA11 mutations is unclear, and further functional experiments are needed to shed light on this.

Our result showed that the ANXA11 gene is one of the most frequently mutated genes, which runs just next to SOD1 in Chinese patients with ALS. Patients in our cohort with ANXA11 mutations showed varied disease characteristics, but no phenotype-genotype correlations were observed. A canonical splice site mutation leading to skipping of the entire exon 6 further supports the loss-of-function mechanism. In addition, our findings further expanded the ANXA11 phenotype, first highlighting its pathogenic role in ALS-FTD.

\section{Author contributions}

Kang Zhang, Qing Liu, Xue Zhang, and Liying Cui designed the experiments. Kang Zhang, Qing Liu, Keqiang Liu, Dongchao Shen, Hongfei Tai, Shi Shu, Hanhui Fu, Shuangwu Liu, and Zhili Wang performed the experiments. Qing Liu, Qingyun Ding, Xiaoguang Li, Mingsheng Liu, and Liying Cui recruited and evaluated the patients. Kang Zhang, Qing Liu, Keqiang Liu, Xue Zhang, and Liying Cui contributed to data analysis and manuscript preparation.

\section{Acknowledgment}

The authors thank all the patients and their families for their cooperation in this study.

\section{Study funding}

This study was funded by the National Key Research and Development Program of China (grant nos. 2016YFC0905100 and 2016YFC0905103); the Chinese Academy of Medical Sciences (CAMS) Innovation Fund for Medical Sciences (CIFMS) (grant nos. 2016-I2M-1-002 and 2016-I2M-1-004); the National Natural Science Foundation of China (NSFC) (grant no. 81230015); and the Beijing Municipal Science and Technology Commission (grant no. Z151100003915078).

\section{Disclosure}

The authors report no disclosures. Full disclosure form information provided by the authors is available with the full text of this article at Neurology.org/NG.

Received January 8, 2018. Accepted in final form March 22, 2018.

\section{References}

1. Taylor JP, Brown RH Jr, Cleveland DW. Decoding ALS: from genes to mechanism. Nature 2016;539:197-206.

2. Andersen PM, Al-Chalabi A. Clinical genetics of amyotrophic lateral sclerosis: what do we really know? Nat Rev Neurol 2011;7:603-615.

3. Brown RH, Al-Chalabi A. Amyotrophic lateral sclerosis. N Engl J Med 2017;377: 162-172.

4. Smith BN, Topp SD, Fallini C, et al. Mutations in the vesicular trafficking protein annexin A11 are associated with amyotrophic lateral sclerosis. Sci Transl Med 2017;9: eaad9157.

5. Brooks BR, Miller RG, Swash M, Munsat TL; World Federation of Neurology Research Group on Motor Neuron Diseases. El Escorial revisited: revised criteria for the diagnosis of amyotrophic lateral sclerosis. Amyotroph Lateral Scler Other Motor Neuron Disord 2000;1:293-299.

6. Neary D, Snowden JS, Gustafson L, et al. Frontotemporal lobar degeneration a consensus on clinical diagnostic criteria. Neurology 1998;51:1546-1554.

7. Rascovsky K, Hodges JR, Knopman D, et al. Sensitivity of revised diagnostic criteria for the behavioural variant of frontotemporal dementia. Brain 2011;134: 2456-2477.

8. Liu Q, Liu F, Cui B, et al. Mutation spectrum of Chinese patients with familial and sporadic amyotrophic lateral sclerosis. J Neurol Neurosurg Psychiatry 2016;87: $1272-1274$.

9. Johnson JO, Pioro EP, Boehringer A, et al. Mutations in the Matrin 3 gene cause familial amyotrophic lateral sclerosis. Nat Neurosci 2014;17:664-666.

10. Smith BN, Ticozzi N, Fallini C, et al. Exome-wide rare variant analysis identifies TUBA4A mutations associated with familial ALS. Neuron 2014;84:324-331.

11. Bannwarth S, Ait-El-Mkadem S, Chaussenot A, et al. A mitochondrial origin for frontotemporal dementia and amyotrophic lateral sclerosis through $\mathrm{CHCHD} 10$ involvement. Brain 2014;137:2329-2345.

12. Kaneb HM, Folkmann AW, Belzil VV, et al. Deleterious mutations in the essential mRNA metabolism factor, hGle1, in amyotrophic lateral sclerosis. Hum Mol Genet 2015;24:1363-1373.

13. Cirulli ET, Lasseigne BN, Petrovski S, et al. Exome sequencing in amyotrophic lateral sclerosis identifies risk genes and pathways. Science 2015;347:1436-1441.

14. Freischmidt A, Wieland T, Richter B, et al. Haploinsufficiency of TBK1 causes familial ALS and fronto-temporal dementia. Nat Neurosci 2015;18:631-636.

15. Brenner D, Muller K, Wieland T, et al. NEK1 mutations in familial amyotrophic lateral sclerosis. Brain 2016;139:e28.

16. Williams KL, Topp S, Yang S, et al. CCNF mutations in amyotrophic lateral sclerosis and frontotemporal dementia. Nat Commun 2016;7:11253.

17. Zou ZY, Zhou ZR, Che CH, Liu CY, He RL, Huang HP. Genetic epidemiology of amyotrophic lateral sclerosis: a systematic review and meta-analysis. J Neurol Neurosurg Psychiatry 2017;88:540-549.

18. Kobayashi Y, Yang S, Nykamp K, Garcia J, Lincoln SE, Topper SE. Pathogenic variant burden in the ExAC database: an empirical approach to evaluating population data for clinical variant interpretation. Genome Med 2017;9:13.

19. Hofmann S, Franke A, Fischer A, et al. Genome-wide association study identifies ANXA11 as a new susceptibility locus for sarcoidosis. Nat Genet 2008;40: 1103-1106.

20. Feng X, Zang S, Yang Y, et al. Annexin A11 (ANXA11) gene polymorphisms are associated with sarcoidosis in a Han Chinese population: a case-control study. BM] Open 2014;4:e004466. 
21. van der Zee J, Gijselinck I, Van Mossevelde S, et al. TBK1 mutation spectrum in an extended european patient cohort with frontotemporal dementia and amyotrophic lateral sclerosis. Hum Mutat 2017;38:297-309.

22. DeJesus-Hernandez M, Mackenzie IR, Boeve BF, et al. Expanded GGGGCC hexanucleotide repeat in noncoding region of C9ORF72 causes chromosome 9p-linked FTD and ALS. Neuron 2011;72:245-256.

23. Renton AE, Majounie E, Waite A, et al. A hexanucleotide repeat expansion in C9ORF72 is the cause of chromosome 9p21-linked ALS-FTD. Neuron 2011;72: 257-268.

24. Kabashi E, Valdmanis PN, Dion P, et al. TARDBP mutations in individuals with sporadic and familial amyotrophic lateral sclerosis. Nat Genet 2008;40: 572-574.
25. Sreedharan J, Blair IP, Tripathi VB, et al. TDP-43 mutations in familial and sporadic amyotrophic lateral sclerosis. Science 2008;319:1668-1672.

26. Crook A, Williams K, Adams L, Blair I, Rowe DB. Predictive genetic testing for amyotrophic lateral sclerosis and frontotemporal dementia: genetic counselling considerations. Amyotroph Lateral Scler Frontotemporal Degener 2017;18:475-485.

27. Wang J, Guo C, Liu S, et al. Annexin A11 in disease. Clin Chim Acta 2014;431: 164-168.

28. Lesniak W, Slomnicki LP, Filipek A. S100A6-new facts and features. Biochem Biophys Res Commun 2009;390:1087-1092.

29. Fukushima T, Zapata JM, Singha NC, et al. Critical function for SIP, a ubiquitin E3 ligase component of the beta-catenin degradation pathway, for thymocyte development and G1 checkpoint. Immunity 2006;24:29-39. 


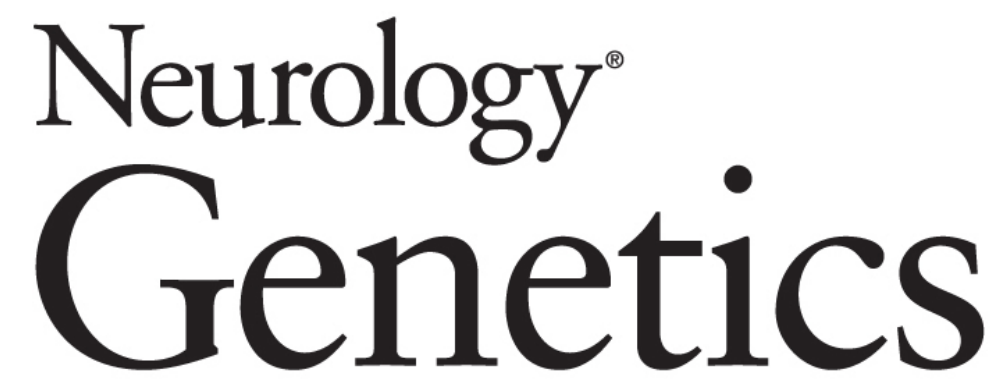

ANXA11 mutations prevail in Chinese ALS patients with and without cognitive

Kang Zhang, Qing Liu, Keqiang Liu, et al. Neurol Genet 2018;4;

DOI 10.1212/NXG.0000000000000237

This information is current as of May 22, 2018

Neurol Genet is an official journal of the American Academy of Neurology. Published since April 2015, it is an open-access, online-only, continuous publication journal. Copyright Copyright ( 2018 The Author(s). Published by Wolters Kluwer Health, Inc. on behalf of the American Academy of Neurology.. All rights reserved. Online ISSN: 2376-7839.

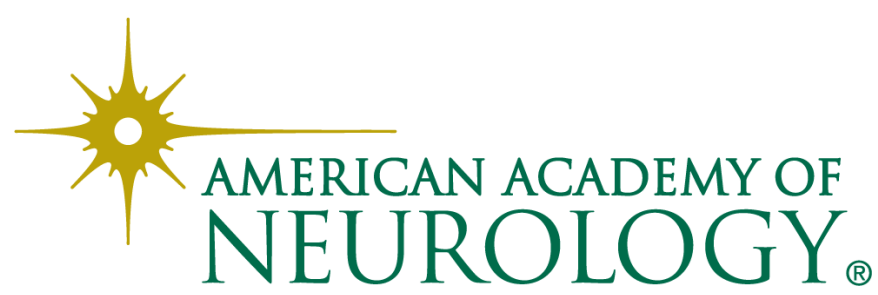




\section{Updated Information \& Services}

References

Citations

Subspecialty Collections

Permissions \& Licensing

Reprints including high resolution figures, can be found at: http://ng.neurology.org/content/4/3/e237.full.html

This article cites 29 articles, 6 of which you can access for free at: http://ng.neurology.org/content/4/3/e237.full.html\#\#ref-list-1

This article has been cited by 4 HighWire-hosted articles: http://ng.neurology.org/content/4/3/e237.full.html\#\#otherarticles

This article, along with others on similar topics, appears in the following collection(s):

\section{All Genetics}

http://ng.neurology.org//cgi/collection/all_genetics

Amyotrophic lateral sclerosis

http://ng.neurology.org//cgi/collection/amyotrophic_lateral_sclerosis_

Frontotemporal dementia

http://ng.neurology.org//cgi/collection/frontotemporal_dementia

Gene expression studies

http://ng.neurology.org//cgi/collection/gene_expression_studies

Peripheral neuropathy

http://ng.neurology.org//cgi/collection/peripheral_neuropathy

Information about reproducing this article in parts (figures,tables) or in its entirety can be found online at:

http://ng.neurology.org/misc/about.xhtml\#permissions

Information about ordering reprints can be found online:

http://ng.neurology.org/misc/addir.xhtml\#reprintsus

Neurol Genet is an official journal of the American Academy of Neurology. Published since April 2015, it is an open-access, online-only, continuous publication journal. Copyright Copyright ( 2018 The Author(s). Published by Wolters Kluwer Health, Inc. on behalf of the American Academy of Neurology.. All rights reserved. Online ISSN: 2376-7839.

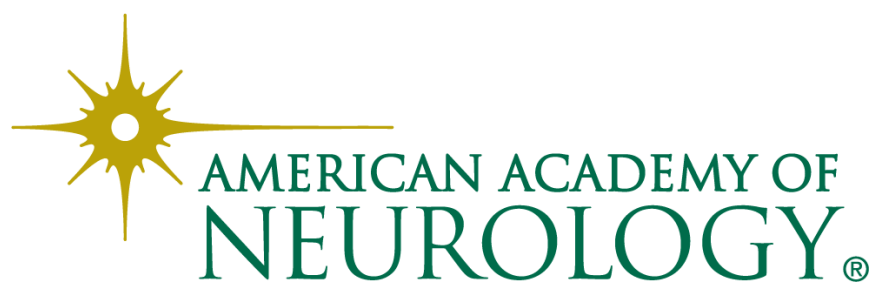

